\title{
Algorithmic aspects of alternating sum of volumes. Part 2: Nonconvergence and its remedy
}

$\mathrm{K}$ Tang and $\mathrm{T}$ Woo

\begin{abstract}
The paper is the second part of a 2-part paper The first part focused on the issues of data structure and fast difference operation. The second studies the nonconvergence of the alternating sum of volumes (ASV) process. An ASV is a series of convex components joined by alternating union and difference operations it is desirable that an ASV series be finite However, such is not always the case - the ASV algorithm can be nonconvergent The paper investigates the causes of this nonconvergence, and finds and proves the conditions responsible for it Linear tıme algorithms are then developed for detection
\end{abstract}

feature extraction, representation conversion, convex hull, alternating sum, difference operatıon, nonconvergence, supportability

An alternatıng sum of volumes (ASV) series is convergent if a deficiency $\boldsymbol{\Omega}_{n}$ is the null set; otherwise, It is said to be nonconvergent (For the computation of efficiency, the detection of a null deficiency $\boldsymbol{\Omega}_{n}$ can be replaced by the determination of the convexity of $\boldsymbol{\Omega}_{n-1}$ ) Figure 1 Illustrates a nonconvergent ASV series. The series of deficiencies $\boldsymbol{\Omega}_{1}, \boldsymbol{\Omega}_{2}, \ldots$, as derived from the convex hull $(\mathrm{CH})$ and difference $(-)$ operations, never converges to the null set, resulting in an infinite alternatıng series. $\left\{\mathrm{CH}(\boldsymbol{\Omega})-\mathrm{CH}\left(\boldsymbol{\Omega}_{1}\right)+\mathrm{CH}\left(\boldsymbol{\Omega}_{2}\right)-\cdots\right.$ $\left.-\mathrm{CH}\left(\boldsymbol{\Omega}_{2 !-1}\right)+\mathrm{CH}\left(\boldsymbol{\Omega}_{2 !}\right)-\cdot\right\}$

As implied in Figure 1, the nonconvergence of an ASV series is determıned by the nonconvergence of a deficiency in its expansion it is known ${ }^{1}$ that an ASV series is nonconvergent when the convex hull of a deficiency $\boldsymbol{\Omega}$, is identified with the convex hull of the deficiency of $\boldsymbol{\Omega}_{i+1}$ For the example shown in Figure 1, the convex hull $\mathrm{CH}\left(\boldsymbol{\Omega}_{1}\right)$ is equal to the convex hull $\mathrm{CH}\left(\boldsymbol{\Omega}_{2}\right)$ As a result of the identification $\mathrm{CH}\left(\boldsymbol{\Omega}_{i}\right)=\mathrm{CH}\left(\boldsymbol{\Omega}_{t+1}\right)$, the following relationship between the deficiencies holds $\boldsymbol{\Omega}_{\boldsymbol{1}}=\boldsymbol{\Omega}_{1+2}(1 \leqslant \jmath)$.

Formally, a deficiency $\boldsymbol{\Omega}_{i}$ is said to be nonconvergent

Department of Industrial and Operations Engineering, Universıty of Michigan, Ann Arbor, Ml 48109-2117, USA

Paper received 13 June 1989 Revised 9 March 1990

If the convex hull of its deficiency $\mathrm{CH}\left(\boldsymbol{\Omega}_{1}\right)-\boldsymbol{\Omega}_{\text {, is equal }}$ to $\mathrm{CH}\left(\boldsymbol{\Omega}_{l}\right)$, and convergent otherwise It is desirable to be able to characterize the nonconvergence of a deficiency $\boldsymbol{\Omega}$, directly, rather than invoke the comparison between $\mathrm{CH}\left(\boldsymbol{\Omega}_{i}\right)$ and $\mathrm{CH}\left(\boldsymbol{\Omega}_{i}\right)-\boldsymbol{\Omega}_{i}$ This pursuit is justified in two respects. First, four convex-hull operations and two set-difference operations must be performed to obtain the datum $\mathrm{CH}\left(\boldsymbol{\Omega}_{l}\right), \mathrm{CH}\left(\boldsymbol{\Omega}_{l}\right)-\boldsymbol{\Omega}_{\text {, }}$ and $\mathrm{CH}\left(\mathrm{CH}\left(\boldsymbol{\Omega}_{i}\right)-\boldsymbol{\Omega}_{i}\right)$ for the comparison. Setdifference operation on a polyhedron with $m$ vertices is known to take at least $O\left(\mathrm{~m}^{2}\right)$ tıme prior to the $O(m \log m)$ result given in Part 1 of this paper ${ }^{2}$. Second, even if the fast $O(m \log m)$ difference operation is Involved, detecting the presence of a null set, as the result of the difference, can be numerically unstable A fast nonconvergence detection algorithm for a

$\Omega$

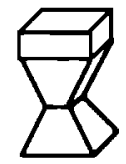

$\Omega_{1}$
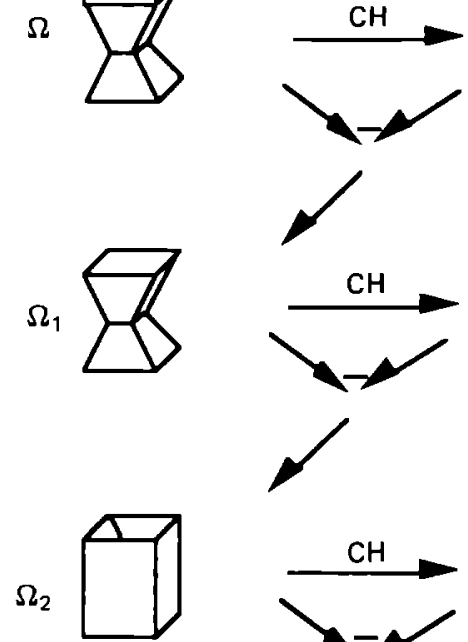

$\Omega_{3}$
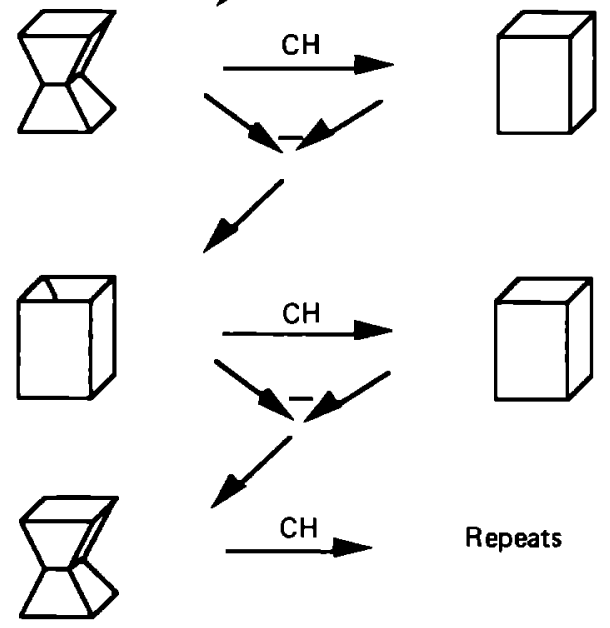

$\mathrm{CH}\left(\Omega_{1}\right)$

Figure 1 Illustration of ASV nonconvergence [CH convex-hull operation, - difference operation ] 
pseudopolyhedron, where the set-difference and comparison operations are not carried out, is a new result in this Part 2 of the paper

Suppose that a fast nonconvergence detection algorithm for a deficiency is avaılable One way in which to detect the nonconvergence of an ASV series is to test for the nonconvergence of every deficiency as it is being computed The time required by such a detection scheme is heavily dependent on the depth $n$ of the first nonconvergent deficiency $\boldsymbol{\Omega}_{n}$ - the larger the number $n$, the more time it will take Alternatively, It may be asked whether the nonconvergence of a series can be detected without invokıng the ASV process itself - not only because the deficiencies thus produced are nonproductive it the ASV series does not converge, but also because a separate scheme may speed up the detection time From the theoretical point of view, such a study addresses some interestıng problems, such as that of findıng the minımum number of faces in a nonconvergent deficiency

These two closely related issues, fast detection of the nonconvergence of a deficiency and that of an ASV series, are investigated in this paper in the next section, the concepts of strong-hull and weak-hull vertices are introduced The characterization of these two types of vertices leads to an $O(n \log n)$ algorithm for detecting the nonconvergence of a deficiency, where $n$ is the number of vertices in the deficiency in the third section of the paper, a sufficient condition for the nonconvergence of an ASV series that requires only lınear tıme to detect is given

\section{CHARACTERIZATION OF NONCONVERGENT DEFICIENCIES}

The following problem is solved in this section given a pseudopolyhedron $\boldsymbol{\Omega}_{\text {, }}$ under what condition will the equation $\mathrm{CH}\left(\boldsymbol{\Omega}_{t}\right)=\mathrm{CH}\left(\mathrm{CH}\left(\boldsymbol{\Omega}_{t}\right)-\boldsymbol{\Omega}_{l}\right)$ hold, and how fast can such a condition be detected? The symbols $\mathrm{CH}$ and - represent the convex hull and regularized difference operations, respectively (Note that every deficiency in an ASV series must be a pseudopolyhedron, as shown in Part 1 of this paper Hereafter, the two terms 'pseudopolyhedron' and 'deficiency' will be used interchangeably) Before the condition for nonconvergence is characterized, it is useful to summarize the relationships between the boundary and interior points of a pseudopolyhedron $\boldsymbol{\Omega}$, its convex hull $\mathrm{CH}(\boldsymbol{\Omega})$, and its deficiency $\mathrm{CH}\left(\boldsymbol{\Omega}_{i}\right)-\boldsymbol{\Omega}$, The first relationshıp, given in Part 1 of this paper, is recited below

Lemma 1 The deficiency of a pseudopolyhedron $\boldsymbol{\Omega}$ is also a pseudopolyhedron, whose interior $\mathbf{I}\left(\mathrm{CH}\left(\boldsymbol{\Omega}_{,}\right)-\boldsymbol{\Omega}_{\boldsymbol{i}}\right)$ Is the set difference $\left\{\mathbf{I}\left(\mathrm{CH}\left(\boldsymbol{\Omega}_{1}\right)\right)-\mathbf{I}\left(\boldsymbol{\Omega}_{1}\right)\right\}$, and the boundary $\mathbf{B}\left(\mathrm{CH}\left(\boldsymbol{\Omega}_{1}\right)-\boldsymbol{\Omega}_{1}\right)$ is a subset of $\left\{\boldsymbol{B}\left(\mathrm{CH}\left(\boldsymbol{\Omega}_{1}\right)\right)-\mathbf{B}\left(\boldsymbol{\Omega}_{t}\right)\right\}$ that forms the closure of $\left\{\mathbf{I}(\mathrm{CH}(\boldsymbol{\Omega}))-,\mathbf{I}\left(\boldsymbol{\Omega}_{1}\right)\right\}$

A pseudopolyhedron is completely described by its faces, and a face is determined by its edges, which are themselves defined by their end points, called vertıces As the set of the vertices of the convex hull of a set of points must be a subset of that point set, by Lemma 1 , the set of the vertices of the deficiency of $\boldsymbol{\Omega}$, is a subset of the vertices of $\boldsymbol{\Omega}$, In other words, the difference operation in the ASV expansion can be viewed as a vertex-elimination process after each difference operation, the deficiency $\boldsymbol{\Omega}$, possesses fewer vertices than does the deficiency $\boldsymbol{\Omega}_{1}$, , this process continues unt I a convex pseudopolyhedron $\boldsymbol{\Omega}_{n}$ is reached whose deficiency $\boldsymbol{\Omega}_{n+1}$ is the null set

If the vertices in the deficiencies cannot be elımınated through the difference operation, the ASV series does not converge A vertex of a pseudopolyhedron $\boldsymbol{\Omega}_{\text {, is }}$ eliminatable if it does not exist in its deficiency $\mathrm{CH}\left(\boldsymbol{\Omega}_{\imath}\right)-\boldsymbol{\Omega}_{\imath}$, otherwise, it is noneliminatable A formal definition of the nonconvergence of the pseudopolyhedron is then in order

Definition 1 A pseudopolyhedron $\boldsymbol{\Omega}$, is nonconvergent if all of its vertices are nonelımınatable, otherwise, it is convergent

To characterıze the elımınatabılıty of vertıces in $\boldsymbol{\Omega}_{\text {, the }}$ vertices are categorized into two groups, hull vertıces and internal vertices The hull vertices are those that are on the boundary of $\mathrm{CH}\left(\boldsymbol{\Omega}_{l}\right)$, whereas those vertices of $\boldsymbol{\Omega}$, that are not on the boundary of $\mathrm{CH}\left(\boldsymbol{\Omega}_{\text {, }}\right)$ are internal. Each of the internal vertices has a 3D neighborhood that is strictly inside $\mathrm{CH}\left(\boldsymbol{\Omega}_{i}\right)$ Further, this neighborhood contains a subset of $\left\{\mathbf{I}\left(\mathrm{CH}\left(\boldsymbol{\Omega}_{,}\right)\right)-\mathbf{I}\left(\boldsymbol{\Omega}_{1}\right)\right\}$, as an internal vertex is also a boundary point of $\boldsymbol{\Omega}$. Therefore, by Lemma 1, all the internal vertices are nonelımınatable To study the elımınatabılity of the hull vertıces, they are further separated into weak and strong hull vertices

Definition 2 In $E^{3}$, the 3D Euclidean space, a hull vertex of $\boldsymbol{\Omega}_{1}$ is weak if it has a 3D neighborhood that contains points in $\left\{\boldsymbol{\Omega}, \cup\left\{\mathrm{E}^{3}-\mathrm{CH}(\boldsymbol{\Omega}),\right\}\right\}$ only, otherwise, it is called a strong hull vertex

As shown in Figure 2, after a difference operation, strong hull and internal vertices remain, whereas all the weak hull vertıces are elımınated Let those faces (edges) of a pseudopolyhedron $\boldsymbol{\Omega}$, be called hull faces (hull edges) If they are completely on the boundary surface of $\mathrm{CH}\left(\boldsymbol{\Omega}_{1}\right)$, and internal taces (internal edges) otherwise Referring to Figure 2, it can be inferred that a hull vertex Is weak if and only if all of its incident faces are hull faces of $\boldsymbol{\Omega}$, (Note, however, that this condition does not hold for incident edges That is, a hull vertex with incident hull edges only is not necessarily weak, as shown in Figure 3, where the strong hull vertex $v$ has no incident internal edges ) The contribution of strong hull vertices to the nonconvergence is demonstrated by the following lemma
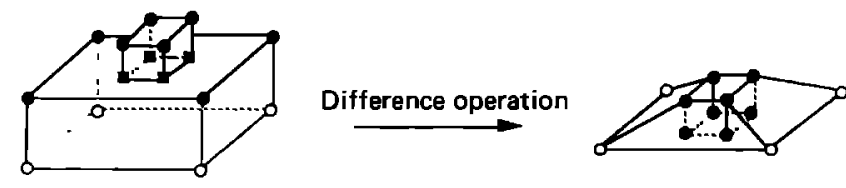

Figure 2. Weak hull vertıces, strong hull vertices and internal vertices

[O weak hull vertıces, strong hull vertıces, $x$ internal vertıces ] 


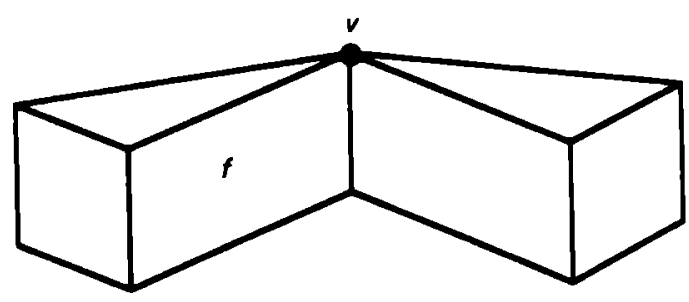

Figure 3 Strong hull vertex with no incident internal edges

Lemma 2. A pseudopolyhedron $\boldsymbol{\Omega}$, is nonconvergent if and only if all of its hull vertices are strong.

Proof First, it is noted that the hull and internal vertices partition the entıre vertex set of $\boldsymbol{\Omega}_{t}$, owing to their mutual exclusıvity By Definition 2, a weak hull vertex has an open $3 D$ neighborhood withın which $\boldsymbol{\Omega}$, is equal to $\mathrm{CH}\left(\boldsymbol{\Omega}_{1}\right)$, and thus there is no subset of $\left\{\mathbf{I}\left(\mathrm{CH}\left(\boldsymbol{\Omega}_{1}\right)\right)-\mathbf{I}\left(\boldsymbol{\Omega}_{1}\right)\right\}$ in that neighborhood Hence, by Lemma 1 , all the weak hull vertıces are elimınatable Conversely, as every 3D neighborhood of a strong hull vertex contains a subset of $\left\{\mathbf{I}\left(\mathrm{CH}\left(\boldsymbol{\Omega}_{1}\right)\right)-\mathbf{I}\left(\boldsymbol{\Omega}_{1}\right)\right\}$, these are preserved on the deficiency of $\boldsymbol{\Omega}$, i.e they are nonelımınatable By Definition 1 and the fact that all the internal vertices are nonelimınatable, the proof is complete

QED

Lemma 2 implies that the detection of the nonconvergence of a pseudopolyhedron $\boldsymbol{\Omega}$, is equivalent to distınguishıng its strong hull vertices from the weak ones Such a process requires two steps classify the hull and internal faces of $\boldsymbol{\Omega}_{i}$, and then check if $\boldsymbol{\Omega}$, has a vertex that has incident hull faces only. Whether a face is internal can be identified by checking one of its interior points. (Such a point must not be on an edge of the face, as an internal face may have hull edges only, e.g. face $f$ in Figure 3.) $\boldsymbol{\Omega}$, is then nonconvergent if and only if no weak hull vertex exists.

The algorithm given below follows the two steps just described It is assumed that a procedure $\operatorname{HULL}\left(N, V, V_{\text {tag }}\right)$ Is in hand that takes a list $V$ of $N$ points as input, and outputs a property array $V_{\text {tag }}$ such that, if $V_{\text {tag }}(1)$ is 'true', point , in $V$ is a hull vertex of $\mathrm{CH}(V)$, and if it is 'false', an internal vertex.

\section{DETECT algorithm}

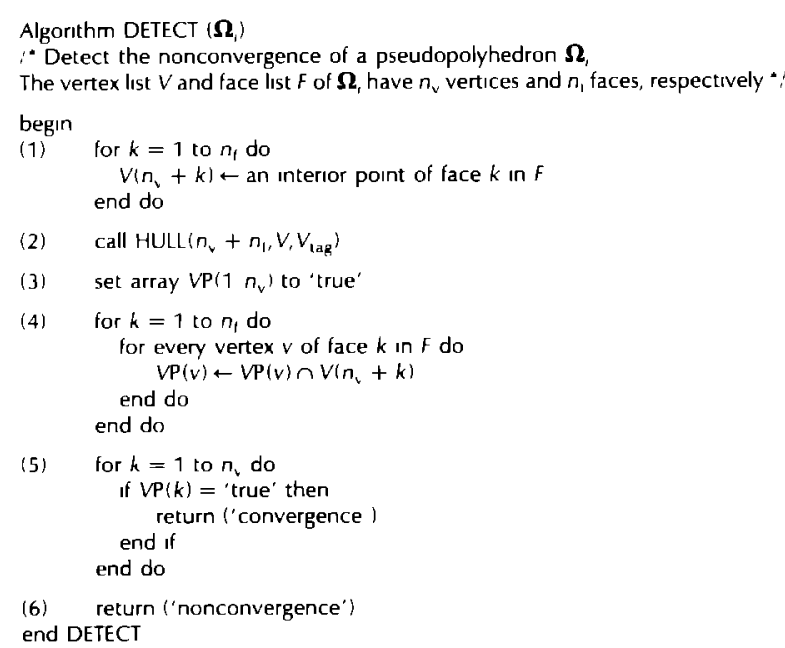

In the DETECT algorithm, the $n_{\mathrm{f}}$ interıor points of the faces of $\boldsymbol{\Omega}$, are first appended to the vertex array $V$ of $\boldsymbol{\Omega}$. As each interior point of a face can be obtained in constant tıme by considerıng any two adjacent edges of that face, Step 1 takes $O\left(n_{\mathrm{f}}\right)$ time. The convex-hull procedure HULL is called at Step 2, which requires only $O\left(\left(n_{v}+n_{f}\right) \log \left(n_{v}+n_{f}\right)\right)$ time $^{3}$ At Step 3, a property array $V P\left(1: n_{v}\right)$ is preset to 'true'. At Step 4, the following is carried out if a face $k$ is internal, i.e. its interıor point $\operatorname{tag} V_{\text {tag }}\left(n_{v}+k\right)$ is 'false', the corresponding entries in $V P$ for all the vertices of face $k$ are reset to 'false' Such a process obviously takes $O(D)$ time, where $D=\Sigma d$, $\left(1=1,2, \ldots, n_{v}\right)$, and $d_{1}$ is the degree of vertex $ı$ it is shown in the Appendix of Part 1 of this paper that $D$ is $O\left(n_{\mathrm{f}}\right)$. Finally, at Step 5 , the array $V \mathbf{P}$ is scanned, and $\boldsymbol{\Omega}$, is identified as convergent if some entry in VP is 'true', and as nonconvergent otherwise. The tıme complexity of the DETECT algorithm is summarized by the following theorem.

Theorem 1 The detection of the nonconvergence of a pseudopolyhedron $\boldsymbol{\Omega}$, with $n$ vertices can be done in $O(n \log n)$ tıme.

Compared with the simple comparison method ${ }^{1}$, $\mathrm{CH}\left(\boldsymbol{\Omega}_{1}\right)=\mathrm{CH}\left(\mathrm{CH}\left(\boldsymbol{\Omega}_{,}\right)-\boldsymbol{\Omega},\right)$, the new detection algorithm DETECT avoıds both the time-consumıng difference operation and the identification of a null set that could be numerically unstable Two convex-hull operations are also saved

It may be noted that the detection algorithm DETECT disregards the disconnectedness of a set The pseudopolyhedron $\boldsymbol{\Omega}$, in Figure 4(a) is nonconvergent, by Lemma 2 The deficiency $\boldsymbol{\Omega}_{t+1}$, however, consists of two separate pseudopolyhedra $\mathbf{P}_{1}$ and $\mathbf{P}_{2}$. Although $\boldsymbol{\Omega}_{t+1}$ is nonconvergent as a single set, it is convergent If represented as $\operatorname{ASV}\left(\boldsymbol{\Omega}_{1+1}\right)=\operatorname{ASV}\left(\mathbf{P}_{1}+\mathbf{P}_{2}\right)=$ $\operatorname{ASV}\left(\mathbf{P}_{1}\right)+\operatorname{ASV}\left(\mathbf{P}_{2}\right)$, because $\mathbf{P}_{1}$ and $\mathbf{P}_{2}$ are both convergent it results in a convergent $\operatorname{ASV}$ tree $\operatorname{ASV}\left(\boldsymbol{\Omega}_{t}\right)=\mathbf{H}-\boldsymbol{\Omega}_{t+1}=\mathbf{H}-\operatorname{ASV}\left(\boldsymbol{\Omega}_{t+1}\right)=$ $\mathbf{H}-\left(\operatorname{ASV}\left(\mathbf{P}_{1}\right)+\operatorname{ASV}\left(\mathbf{P}_{2}\right)\right)$, which branches at the deficiency $\boldsymbol{\Omega}_{\imath+1}$ In some other cases, a pseudopolyhedron, though connected, might be separated uniquely at some edges such that the separated subsets
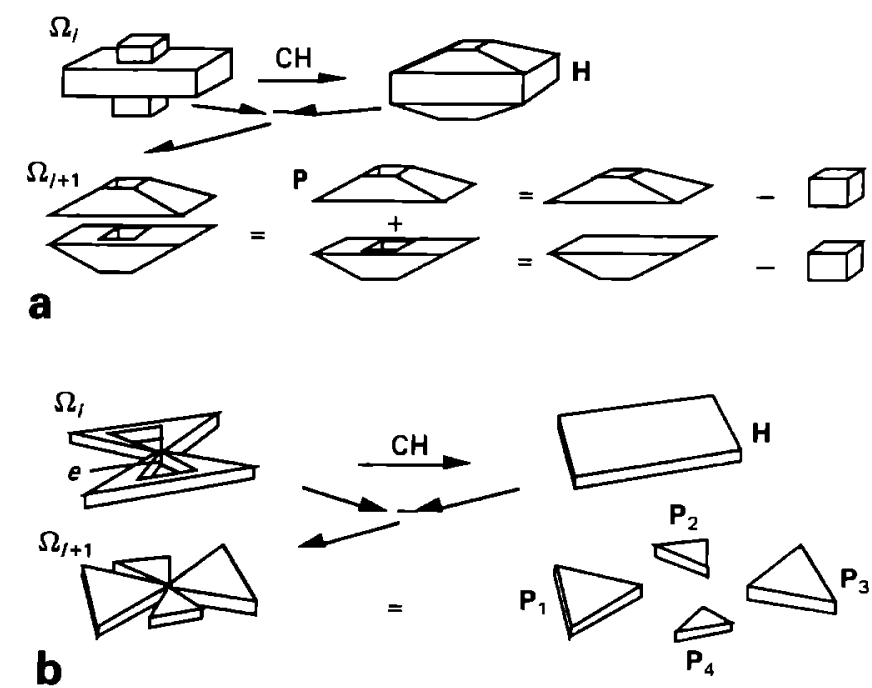

Figure 4 Convergence by set separation 


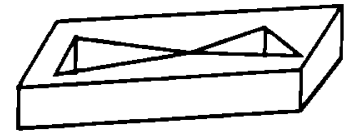

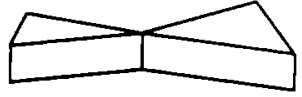

b
Figure 5 Well connected set versus III connected set, (a) well connected set, (b) Ill connected set

are all convergent For example, the pseudopolyhedron $\boldsymbol{\Omega}$, in Figure 4(b) is nonconvergent, by Lemma 2 It is, however, convergent if expressed as $\boldsymbol{\Omega}_{1}=\mathbf{H}-\boldsymbol{\Omega}_{1+1}=$ $\mathbf{H}-\left(\mathbf{P}_{1}+\mathbf{P}_{2}+\mathbf{P}_{3}+\mathbf{P}_{4}\right)$, as $\mathbf{P}_{1}, \mathbf{P}_{2}, \mathbf{P}_{3}$ and $\mathbf{P}_{4}$ are all convergent.

Both examples of set separation on $\boldsymbol{\Omega}_{t+1}$ shown in Figure 4 have a crucial property the boundary of the separated pseudopolyhedron remains unchanged Unlıke in polyhedral decomposition ${ }^{4}$, such a property guarantees that the boundary after the separation has the same sets of vertices, edges and faces as before, with only the adjacency and incidence relationships between them being altered. Further, it is next shown that such a separation is unique, thus justifyıng the exıstence of a determınıstıc algorıthm To define set separation rigorously, the concept of well connectedness is needed

Definition 3 Two points $p$ and $q$ of a pseudopolyhedron $\boldsymbol{\Omega}$, are said to be well connected in $\boldsymbol{\Omega}$, if there exists a curve $c$ between $p$ and $q$ such that all the points in $c$, except for possibly $p$ and $q$, are in $\mathbf{I}\left(\boldsymbol{\Omega}_{t}\right) \boldsymbol{\Omega}$, is a well connected set if all of its points are well connected in it, otherwise it is an II/ connected set (see Figure 5)

The $\boldsymbol{\Omega}_{i+1}$ in Figure 4(a), and both $\boldsymbol{\Omega}$, and $\boldsymbol{\Omega}_{t+1}$ in Figure 4 (b), are ill connected pseudopolyhedra A well connected pseudopolyhedron is also called a robust set, meanıng that its interior is all connected A subset $\zeta$ of a pseudopolyhedron $\boldsymbol{\Omega}$, is a maximally well connected set (MWCS) of $\boldsymbol{\Omega}$, if $\zeta$ is a well connected set, and any addition of non- $\zeta$ points of $\boldsymbol{\Omega}$, to $\zeta$ will constitute an Ill connected set As an example, only $\mathbf{P}_{1}, \mathbf{P}_{2}, \mathbf{P}_{3}$ and $\mathbf{P}_{4}$ are the MWCSs of the pseudopolyhedron $\boldsymbol{\Omega}_{t+1}$ in Figure 4(b)

It is desirable that an ASV series be expanded as much as possible so that more features can be extracted Once a nonconvergent and Ill connected deficiency is encountered, it should be separated into the MWCSs, and the ASV process can then be performed on each of these This leads to the notion of strong and weak nonconvergence.

Definition 4 A nonconvergent pseudopolyhedron $\boldsymbol{\Omega}_{t}$ is strongly nonconvergent if both itself and its deficiency are robust Otherwise, $\boldsymbol{\Omega}$, is weakly nonconvergent

As examples, the deficiency $\boldsymbol{\Omega}_{1}$ in Figure 1 is strongly nonconvergent, as both it and its deficiency $\boldsymbol{\Omega}_{2}$ are robust, whereas- each $\boldsymbol{\Omega}$, in Figure 4 is weakly nonconvergent, because either it, or its deficiency $\boldsymbol{\Omega}_{1+1}$, is ill connected.

The detection of the strength of nonconvergence of a pseudopolyhedron $\boldsymbol{\Omega}_{\text {, }}$ of $n$ faces requires three steps the identification of its nonconvergence, the computation for the deficiency of $\boldsymbol{\Omega}_{\text {, }}$ and the classification of the well connectedness of $\boldsymbol{\Omega}$, and/or
Its deficiency The first step can be done, by Theorem 1 , in $O(n \log n)$ tıme The difference operation also requires $O(n \log n)$ time, as shown in Part 1 of this paper Thus, it the classification of the robustness of $\boldsymbol{\Omega}$, can be done in $O(n \log n)$ tıme, then so can the detection of the strong nonconvergence. As $\boldsymbol{\Omega}$, is robust if and only if its MWCS separation contains only one MWCS, i e Itself, the goal becomes the finding of an $O(n \log n)$ time MWCS separation algorithm

In implementıng such an algorithm, it is noted that, by the definition of a pseudopolyhedron, the well connectedness of its boundary-point set will ensure that it is a well connected set This fact ensures that the MWCSs of a pseudopolyhedron can be detected by checkıng only the well connectedness of its faces

Let two faces of a pseudopolyhedron be well adjacent to each other if they share a common edge and are well connected to each other It can easily be shown that two faces $A$ and $B$ of a pseudopolyhedron are well connected if and only if etther they are well adjacent, or there exists a number of faces $t_{1}, t_{2}, \ldots, l_{k}$ such that $A$ is well adjacent to $f_{1}, t_{1}$ is well adjacent to $f_{2}$, and $t_{k}$ is well adjacent to $B$ For example, in Figure 6 , faces $A$ and $B$ are not well connected, because the curve c connectıng points $p$ and $q$ passes through edge $e$, which does not belong to the interior of that pseudopolyhedron

To characterize face well adjacency, let $f_{1}, f_{2}, \quad, f_{m}$ be the faces incident to a common edge, ordered by their spatial angles (In Figure 7, $f_{1}, f_{2},, f_{m}$ are the intersections between the faces sharing a common

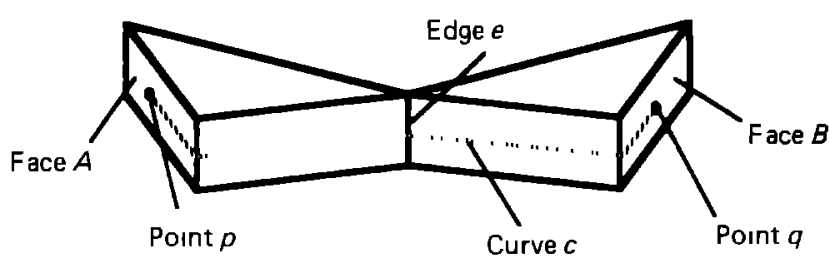

Figure 6 III connectedness of faces of a pseudopolyhedron

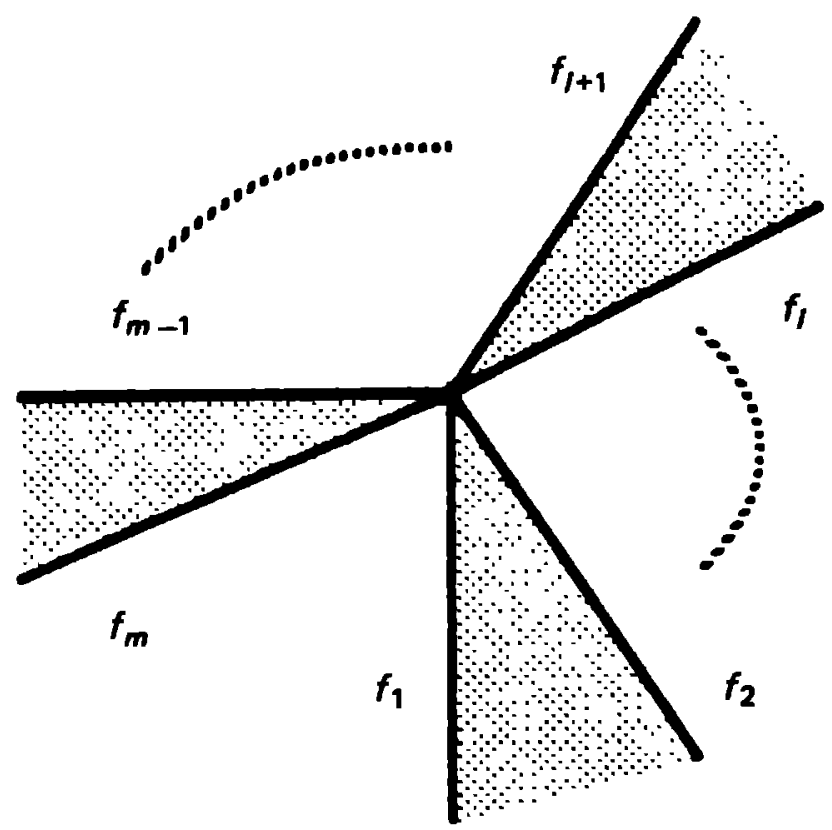

Figure 7 Well adjacency of taces 
edge and a plane orthogonal to that edge ) Apparently, the well adjacent face of face $f_{1}(t=1,2, \ldots, m)$ is either $t_{t-1}$ or $f_{t+1}(\bmod m)$, depending on the direction of the outward normal of $f_{1}$. Such a pairing process can be done in $O(m)$ tıme The following recursive procedure MWCS_FACES finds the faces of a maximally well connected set of a pseudopolyhedron $\boldsymbol{\Omega}$, The Input is the pseudopolyhedron representation $\{V, E, F$, NORM, $\left.E_{f}\right\}$ of $\boldsymbol{\Omega}$, and the index of a face of $\boldsymbol{\Omega}_{1}$. The output is the indices of those faces of $\boldsymbol{\Omega}$, that form the boundary of an MWCS of $\boldsymbol{\Omega}$,

\section{MWCS_FACES procedure}

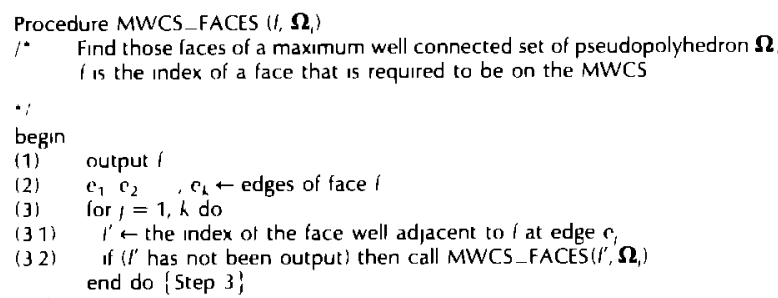

Suppose that a total of $m$ edges $e_{1}, e_{2}, \quad, e_{m}$ of $\boldsymbol{\Omega}$, are found in an MWCS, denoted by $P$, through MWCS_FACES Let $k_{1}$ and $k_{1}^{\prime}$ be the face-adjacency indices of $\boldsymbol{\Omega}$, and $P$ at edge $e_{1}$, respectively $(\iota=1,2, \ldots, m)$ Step 1 takes constant tıme, and thus the overall tıme spent at Step 1 when MWCS_FACES termınates is $O(n)$, where $n$ is the number of faces on $P$. As each face of $P$ is processed only once, the overall tıme required by Step 2 is $O\left(\Sigma\left(k_{1}^{\prime}\right)(ı=1,2, \quad, m)\right)$ As for the loop at Step 3, note that the indices of the faces of $\boldsymbol{\Omega}$, adjacent to edge e, are stored in the order of their spatial angles in an entry of the $E_{f}$ list of $\boldsymbol{\Omega}$, Therefore, only $O\left(\log k_{l}\right)$ time is needed to locate the position of $f$ in that entry, and, hence, the index $f^{\prime}$ of the face well adjacent to face $f$ at an edge $e$, As a result, the overall tıme taken by Step 31 is $O\left(\Sigma\left(k_{\prime}^{\prime} \log k_{\prime}\right)\right.$ $(s=1,2, \quad, m))$ The total time cost of MWCS_FACES Is therefore $O\left(n+\left(\Sigma\left(k_{1}^{\prime} \log k_{1}\right)(l=1,2, ., m)\right)\right)$.

Before the complete algorithm for carrying out the MWCS separation is presented, it is necessary to clarify that, given the indices of $n$ faces that form the boundary of an MWCS of $\boldsymbol{\Omega}_{i}$ only $O(n)$ time is needed to construct the pseudopolyhedron representation $\left\langle V, E, F, N O R M, E_{f}\right\rangle$ of that MWCS, say $P_{i}$. To see this, note that all the $V, E, F, N O R M$ and $E_{\mathrm{f}}$ lists of $P$, are readily available in the $\left\langle V, E, F, N O R M, E_{f}\right\rangle$ of $\boldsymbol{\Omega}$, The only work needed besides the retrieval is the reındexation of the vertices, edges and faces of $P$, once they are retrieved from $\boldsymbol{\Omega}$, For example, if only vertices $\left\{v_{3}, v_{4}, v_{7}, v_{9}, v_{15}\right\}$ are on $P_{1}$, and there is an edge on $P$, whose entry in the $E$ list of $\Omega$, is $\langle 9,4\rangle$, then this edge will become $\langle 4,2\rangle$ in the $E$ list of $P_{1}$, because vertices $v_{9}$ and $v_{4}$ now sit at the fourth and second positions of the $V$ list of $P_{1}$ Analogously, if edges $\left\{e_{2}, e_{7}, e_{10}, e_{13}\right\}$ are on $P_{11}$ and $P$, has a face stored in the $F$ list of $\boldsymbol{\Omega}$, as $\langle 10,7,13\rangle$, then this face will become $\langle 3,2,4\rangle$, owing to the reindexation of $\left\{e_{2}, e_{7}, e_{10}, e_{13}\right\}$. Clearly, this reindexation process can be done in $O(n)$ time through sımple index mapping Let MWCS_OUTPUT be such a process, that takes as input a pseudopolyhedron $\boldsymbol{\Omega}$, and a list $L$ of indices of the faces of $\boldsymbol{\Omega}_{1}$, and outputs the pseudopolyhedron representation of an MWCS of $\boldsymbol{\Omega}$, whose faces are those of $\boldsymbol{\Omega}$, with indices in $L$. Using both procedures MWCS_FACES and MWCS_OUTPUT, the algorithm given next performs the MWCS separation

\section{MWCS_SEPARATION algorithm}

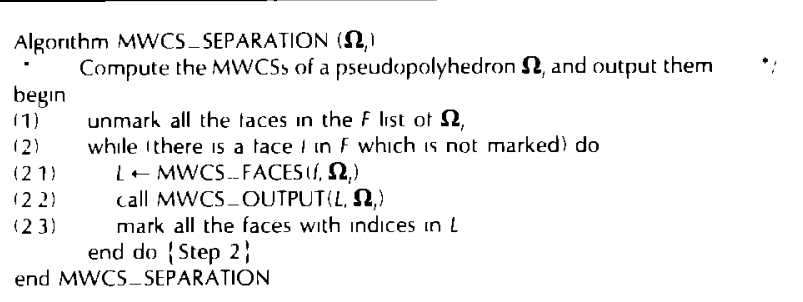

Lemma 3-The MWCS separation of a pseudopolyhedron $\boldsymbol{\Omega}$, with $n_{\mathrm{f}}$ faces can be done in $O\left(n_{\mathrm{f}} \log n_{\mathrm{f}}\right)$ time and $O\left(n_{\mathrm{f}}\right)$ space

Proof. In the MWCS_SEPARATION algorithm, Step 1 takes $O\left(n_{f}\right)$ time. For the while loop at Step 2, as each face can only be in one MWCS, the overall time cost of Step 22 and Step 2.3 is clearly $O\left(n_{\mathrm{f}}\right)$ The tıme taken by each execution of the procedure MWCS_FACES is in the form of $O\left(n+\left(\Sigma\left(k_{\prime}^{\prime} \log k_{\imath}\right)(\imath=1,2, \ldots, m)\right)\right)$, where $n$ and $m$ are the numbers of the faces and edges on that particular MWCS, and $k_{\text {, and }} k_{1}^{\prime}$ are the numbers of the faces of $\boldsymbol{\Omega}$, and that MWCS adjacent to an edge of the MWCS, respectively. For the same reason that a face of $\boldsymbol{\Omega}$, can only be in one of its MWCSs, the sum of $\Sigma k_{1}^{\prime}(l=1,2, \ldots, m)$ over all the edges of $\boldsymbol{\Omega}$, is $O\left(\Sigma k_{\text {, }}\right.$ $\left.\left(l=1,2, \quad, n_{\mathbf{e}}\right)\right)$, where $n_{\mathbf{e}}$ is the total number of edges of $\boldsymbol{\Omega}$. Therefore, after the termınation of the MWCS_SEPARATION algorithm, the overall time taken by Step 21 is $O\left(n_{\mathrm{f}}+\left(\Sigma k_{\mathrm{f}}\right) \log n_{\mathrm{f}}\right)$, i.e. $O\left(n_{\mathrm{f}} \log n_{\mathrm{f}}\right)$, as $\Sigma k_{1}\left(\iota=1,2, \ldots, n_{\mathrm{e}}\right)$ is $O\left(n_{\mathrm{f}}\right)$.

QED

With Theorem 1 and Lemma 3, the following is in order.

Theorem 2 - Whether a pseudopolyhedron $\mathbf{\Omega}$, is strongly nonconvergent or not can be detected in $O(n \log n)$ tıme, where $n$ is the number of faces of $\boldsymbol{\Omega}$,

It Is worth noting that, in the ASV process, the MWCS_SEPARATION algorithm not only detects the strong nonconvergence of a deficiency $\boldsymbol{\Omega}_{1}$, but also constructs the MWCSs of the deficiency $\boldsymbol{\Omega}_{++1}$ The pseudopolyhedron representation of the MWCSs can then be used for the subsequent convex-hull and difference operations, along the corresponding branches after $\boldsymbol{\Omega}_{\imath+1}$.

\section{FAST DETECTION FOR ASV NONCONVERGENCE}

An ASV series is nonconvergent if it has a nonconvergent deficiency $\boldsymbol{\Omega}_{n}$. A way to detect the nonconvergence of an ASV series is to check the nonconvergence of every deficiency in the series. The tıme required by such a detection sets an upper bound

Theorem 3 It needs at most $O\left(n^{2} \log n\right)$ time to decide whether the ASV series of a pseudopolyhedron $\boldsymbol{\Omega}$ is 


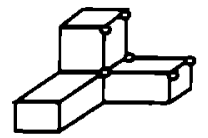

a

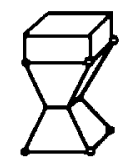

$\cdot$

b

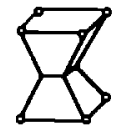

Figure 8. Regularized set intersection

convergent or not, where $n$ is the number of vertices of $\boldsymbol{\Omega}$

Proof Recall that the difference operation is a vertex-elımınation process. That is, a nonconvergent deficiency $\boldsymbol{\Omega}_{k}$ in $\operatorname{ASV}(\boldsymbol{\Omega})$ always has fewer vertices than $\boldsymbol{\Omega}_{k-1}$. In the worst case, suppose that only one vertex is elimınated after each difference operation To obtain the deficiency $\boldsymbol{\Omega}_{k}$ through the ASV process, $k$ convex-hull and difference operations are needed, resultıng in an overall tıme requirement of $\Sigma O\left({ }_{1} \log I_{1}\right)$ $(l=n, n-1, \quad, n-k)$. Therefore, at most $\Sigma O(\iota \log I)$ $(i=n, n-1, \quad, 1) \leqslant O\left(n^{2} \log n\right)$ time is needed to detect whether $\operatorname{ASV}(\boldsymbol{\Omega})$ converges or not.

QED

In an attempt to improve this upper bound, the local cause of the ASV nonconvergence of a pseudopolyhedron $\boldsymbol{\Omega}$ is sought Such a study results in a sufficient condition for the ASV nonconvergence, which eventually leads to a linear detection algorıthm In the search for this local cause, it is useful to invoke the mechanics of regularized intersection ${ }^{5}$

Definition 5 The regularized intersection of two pseudomanifolds $\mathbf{A}$ and $\mathbf{B}$, denoted by $\mathbf{A} * \mathbf{B}$, is a pseudomanifold whose interior is $\mathbf{I}(\mathbf{A}) \cap \mathbf{I}(\mathbf{B})$

Figure 8 gives two examples of regularized intersection. As shown in Figure 8(a), the regularized intersection $\mathbf{A} * \mathbf{B}$ is the null set $\varnothing$, even though the ordinary set intersection $\mathbf{A} \cap \mathbf{B}$ yields two faces The result of the regularized intersection in Figure $8(b)$ is a nonconvergent pseudopolyhedron A tantalızıng finding is revealed by the example in Figure $8(b)$ if there exists a (nonempty) subset (prior to the regularized intersection) that is nonconvergent, then the ASV series to be expanded is nonconvergent Such an observatıon is not a coincidence, the basis of this is shown by the following Lemma 4

Lemma 4. Let $\zeta$ be a subset of the vertices of a pseudopolyhedron $\boldsymbol{\Omega}$. If the regularized intersection between $\boldsymbol{\Omega}$ and $\mathrm{CH}(\zeta)$ is a nonconvergent pseudopolyhedron, the ASV series of $\boldsymbol{\Omega}$ is nonconvergent.

Proof Assume that $\mathbf{\Omega} * \mathrm{CH}(\zeta)$ is a nonconvergent pseudopolyhedron It is claimed that all the vertices in $\zeta$ are noneliminatable Suppose that there is a deficiency $\boldsymbol{\Omega}$, in $\operatorname{ASV}(\boldsymbol{\Omega})$ whose vertex set is a superset of $\zeta$, such that some vertex $v$ in $\zeta$ is lost on the deficiency $\boldsymbol{\Omega}_{1+1}$ By Lemma 2, this means that all the incident faces of $v$ are the hull faces of $\boldsymbol{\Omega}_{1}$ As $\mathrm{CH}(\zeta)$ is a subset of
$\mathrm{CH}(\boldsymbol{\Omega}$, , it follows that $v$ is also a weak hull vertex of $\boldsymbol{\Omega} * \mathrm{CH}(\zeta)$, which contradicts the assumption that $\boldsymbol{\Omega} * \mathrm{CH}(\zeta)$ is nonconvergent

QED

Lemma 4 provides a sufficient condition for the nonconvergence of an ASV series, without the ASV process itself being invoked A direct implementation of such an algorithm is, however, infeasible, as there are $O\left(n^{\prime}\right)$ subsets To reduce this high complexity, the characterization of local subsets of vertices, i e those that are adjacent to a common vertex, is investigated

Let two vertices of a pseudopolyhedron be said to be adjacent to each other if they are the two end points of an edge

Definition 6 A vertex $v$ of a pseudopolyhedron $\boldsymbol{\Omega}$ is supportable if there exısts a plane contaınıng $v$ such that the point set $\xi_{v}$ lies on one side of that plane (where $\xi_{\mathrm{v}}$ consists of those vertices that are adjacent to $v$ ), otherwise, $v$ is a nonsupportable vertex

As an example, all the vertices except for $v$ of the pseudopolyhedron in Figure 9(a) are supportable. Also, as shown in Figure 9(b), a vertex $v$ is nonsupportable If and only if it is strictly inside the convex hull of the vertices adjacent to it

Lemma 5 If a pseudopolyhedron $\boldsymbol{\Omega}$ has a nonsupportable vertex, then the ASV series of $\boldsymbol{\Omega}$ is nonconvergent

Proof Let $v$ be a nonsupportable vertex of $\boldsymbol{\Omega}$, and $\xi_{v}$ be the point set consisting of those vertices that are adjacent to $v$ The lemma is proven by showing that $\boldsymbol{\Omega} * \mathrm{CH}\left(\xi_{\mathrm{v}}\right)$ is a nonconvergent pseudopolyhedron As $v$ Is internal to $\mathrm{CH}\left(\xi_{v}\right)$, all its incident faces have portions that are internal to $\mathrm{CH}\left(\xi_{v}\right)$ Then, in each of these faces, there is a point that has an open 3D neighborhood that contains a subset of $\mathbf{I}(\boldsymbol{\Omega})$ that is strictly inside $\mathrm{CH}\left(\xi_{\mathrm{v}}\right)$. By the definition of the regularized intersection, this neighborhood is preserved on $\boldsymbol{\Omega} * \mathrm{CH}\left(\xi_{\mathrm{v}}\right)$ In other words, $\boldsymbol{\Omega} * \mathrm{CH}\left(\xi_{\mathrm{v}}\right)$ must be a pseudopolyhedron, as its interior is not empty Now, consider a hull vertex $p$ of $\boldsymbol{\Omega} * \mathrm{CH}\left(\xi_{v}\right)$, as illustrated in Figure 10(a) If $p$ belongs to $\xi_{v}$, as none of the incident faces of $v$ can be a hull face of $\mathrm{CH}\left(\xi_{v}\right), p$ can only be a strong hull vertex of $\boldsymbol{\Omega} * \mathrm{CH}\left(\xi_{v}\right)$ If $p$ does not belong to $\xi_{v}$, it must be an intersection point between some face $f$ of $\boldsymbol{\Omega}$ and a hull face of $\mathrm{CH}\left(\xi_{\mathrm{v}}\right)$ (see Figures 10(b) and (c)) As face $f$ has a portion internal to $\mathrm{CH}\left(\xi_{v}\right)$, which becomes an internal face of $\boldsymbol{\Omega} * \mathrm{CH}\left(\xi_{\mathrm{v}}\right), p$ must be a strong hull vertex of $\boldsymbol{\Omega} * \mathrm{CH}\left(\xi_{v}\right)$. Therefore, all the

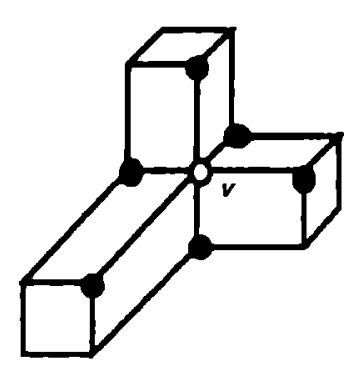

a

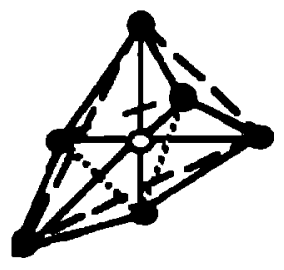

b
Figure 9 Supportable and nonsupportable vertices 


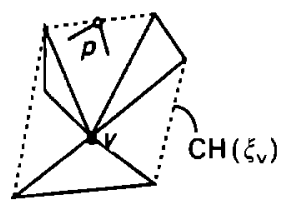

a b

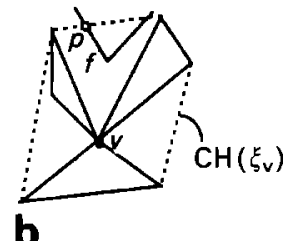

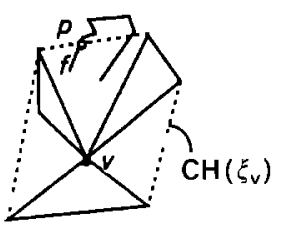

C
Figure 10 Proof of Lemma 6

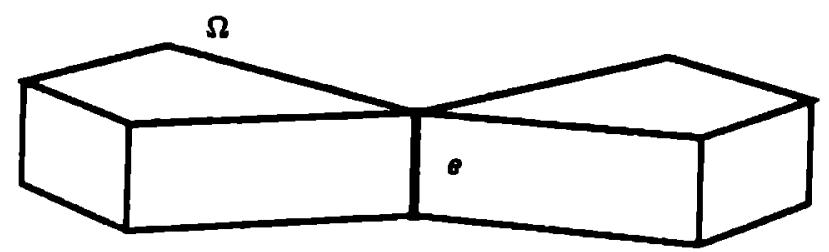

a

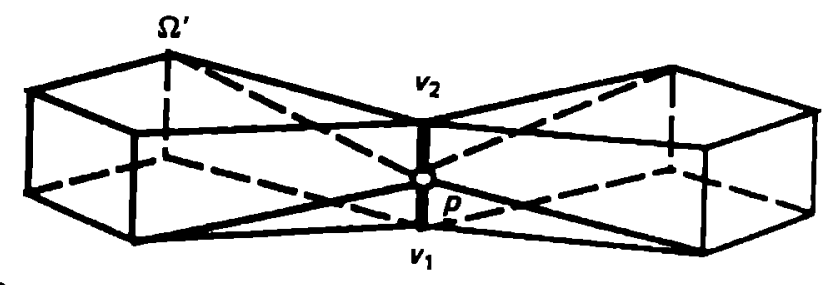

b

Figure 11 Nonsupportable vertex introduced by a nonsupportable edge

vertices of $\boldsymbol{\Omega} * \mathrm{CH}\left(\xi_{\mathrm{v}}\right)$ are either internal or strong. By Lemma $2, \boldsymbol{\Omega} * \mathrm{CH}\left(\xi_{\mathrm{v}}\right)$ is nonconvergent.

QED

As an illustration of Lemma 5, vertex $v$ of the pseudopolyhedron in Figure 9(a) is nonsupportable. The regularized intersection between the pseudopolyhedron and $\mathrm{CH}\left(\xi_{v}\right)$, where $\xi_{v}$ are those six vertices adjacent to $v$, is another pseudopolyhedron, as shown In Figure 9(b), that is nonconvergent By Lemma 5, the ASV series of the pseudopolyhedron in Figure 9(a) does not converge, this can easily be verified

An extension to the supportability of vertices is the supportability of edges. Consıder the pseudopolyhedron $\boldsymbol{\Omega}$ in Figure 11(a). Its ASV series can easily be shown to be nonconvergent, although all its vertices are supportable

Definition 7. An edge e of a pseudopolyhedron is supportable if there exists a plane contaınıng e such that all the faces incident to e are on one side of that plane, otherwise, edge e is nonsupportable

Lemma 6. If pseudopolyhedron $\boldsymbol{\Omega}$ has a nonsupportable edge $e$, then $\operatorname{ASV}(\boldsymbol{\Omega})$ is nonconvergent.

Proof Assume that the nonsupportable edge $\mathrm{e}$ has $k$ Incident faces $f_{1}, f_{2}, \ldots, f_{k}$, and that $v_{1}$ and $v_{2}$ are its two vertices Let $p$ be an arbitrary point on $e$, but not $v_{1}$ or $v_{2}$. Also, let $\rho_{1}$, which is not $v_{1}$ or $v_{2}$, be a vertex on face $f_{i}(l=1,2,, k)$ As the line segment $\left[p, p_{l}\right]$ is

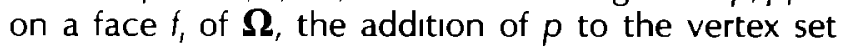
of $\boldsymbol{\Omega}$, and the addition of edges $\left[\rho, v_{1}\right],\left[p, v_{2}\right]$, , and $\left[p, p_{l}\right](l=1,2, \quad, k)$ to the edge set of $\boldsymbol{\Omega}$, introduces a new pseudopolyhedron representation of $\boldsymbol{\Omega}$, as shown in Figure 11(b) As e is nonsupportable, all the points in it, except, possibly, for $v_{1}$ or $v_{2}$, are strictly inside $\mathrm{CH}\left(\left\{v_{1}, v_{2}, p_{1}, p_{2}, \ldots, p_{k}\right\}\right)$. This implies that the vertex $p$ is nonsupportable By Lemma $5, \operatorname{ASV}(\boldsymbol{\Omega})$ is nonconvergent.

QED

It should be mentioned that Lemma 5 and Lemma 6 supply a sufficient but not necessary condition for nonconvergence As an example, all the vertices and edges of the polyhedron in Figure 12 are supportable However, its ASV series is nonconvergent

Nevertheless, a lınear tıme algorithm for detectıng the sufficiency of nonconvergence offers an attractive alternative to the $O\left(n^{2} \log n\right)$ tıme for both necessity and sufficiency

Let $o$ be the origin and $\left(x_{1}, y_{1}, z_{1}\right),\left(x_{2}, y_{2}, z_{2}\right)$, $\left(x_{k}, y_{k}, z_{k}\right)$ be $k$ points in the 3D space If the point $o$ is supportable against $\left(x_{1}, y_{1}, z_{1}\right),\left(x_{2}, y_{2}, z_{2}\right)$, $\left(x_{k}, y_{k}, z_{k}\right)$, the angle between the normal vector $\mathbf{N}_{o}$ of a supportıng plane $P_{0}$ and the vector $\left(x_{1}, y_{1}, z_{1}\right)$ must not be greater than $90^{\circ}$ for all the $i=1,2, \quad, k$ (see Figure 13) Conversely, if there exists a vector $\mathbf{N}_{o}$ such that the angle between it and a vector $\left(x_{1}, y_{1}, z_{1}\right)$ $(1=1,2, \quad k)$ is less than or equal to $90^{\circ}$, then the plane passıng through o and orthogonal to $\mathbf{N}_{o}$ is clearly a supporting plane Therefore, the detection of the supportability becomes the following given $k$ vectors $\left(x_{1}, y_{1}, z_{1}\right),\left(x_{2}, y_{2}, z_{2}\right), \ldots,\left(x_{k}, y_{k}, z_{k}\right)$, find another nonzero vector $(A, B, C)$ such that $A x_{1}+B y_{1}+C z_{1} \geqslant 0$ $(i=1,2, \ldots, k)$ It is known ${ }^{3}$ that the solution to this 3 -variable problem, if it exists, can be obtaıned in $O(k)$ tıme

Let $\operatorname{SUPPORT}(k, L)$ be a supportability detection procedure that takes a list $L$ of $k$ points as input, and outputs either 'true', If the origin is supportable against $L$, or 'false' otherwise With the SUPPORT procedure, the following algorithm is in order.

\section{NSV_DETECT algorithm}

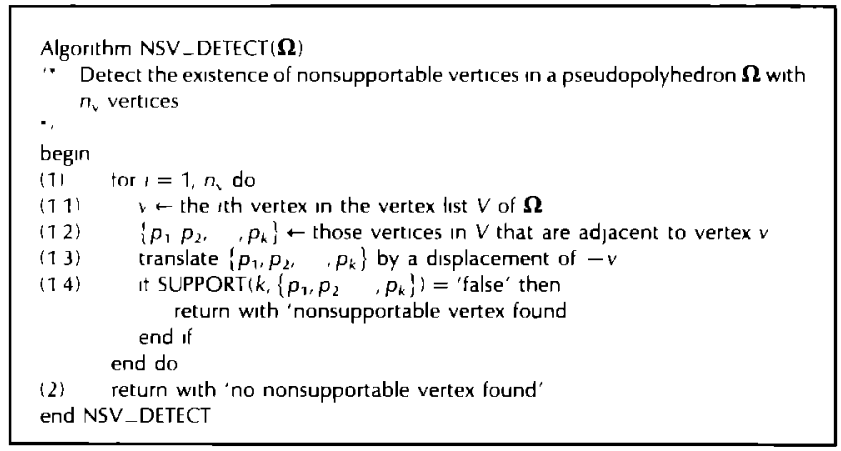

To devise an algorithm for detectıng the supportability of an edge e of a pseudopolyhedron $\boldsymbol{\Omega}$, let $v$ and $v^{\prime}$

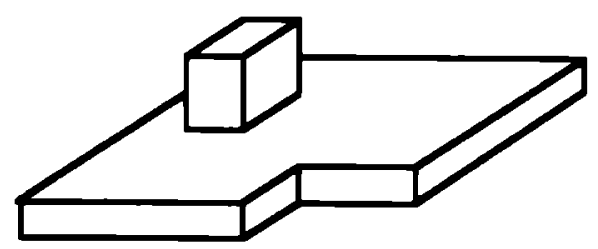

Figure 12. Nonconvergent polyhedron with no nonsupportable vertices or edges 


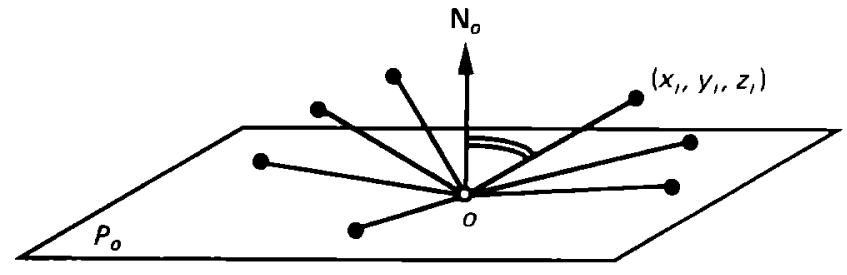

Figure 13. Angular relationship between the normal of a supporting plane and the adjacent vertices

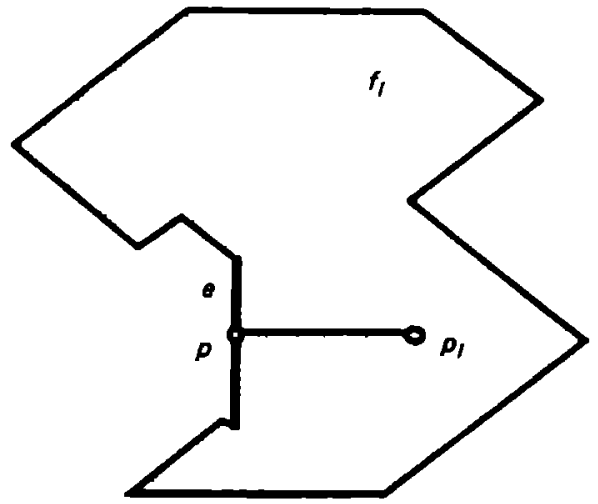

Figure 14 Finding the point $p$, on face $f$

be the two end points of e, $p$ be its center point, and $f_{1}, f_{2}, \quad, f_{k}$ be the faces of $\boldsymbol{\Omega}$ incident to e Also, let $p_{i}$ be a point on face $f$, such that the line segment $\left[p, p_{1}\right]$ completely belongs to $f_{1}(t=1,2, \quad, k)$ (see Figure 14). Such a point $p$, can be obtained in the constant time from the (clockwise or counterclockwise) order of the edges Let $\mathrm{FS}\left(p, f_{1}\right)$ denote the function that returns the point $p_{l}$. Referring to the proof of Lemma 6 , e is supportable if and only if $p$ is supportable against the point set $\left\{v, v^{\prime}, p_{1}, p_{2}, p_{k}\right\}$. This equivalence relatıonshıp gives rise to the following algorithm

\section{NSE_DETECT algorithm}

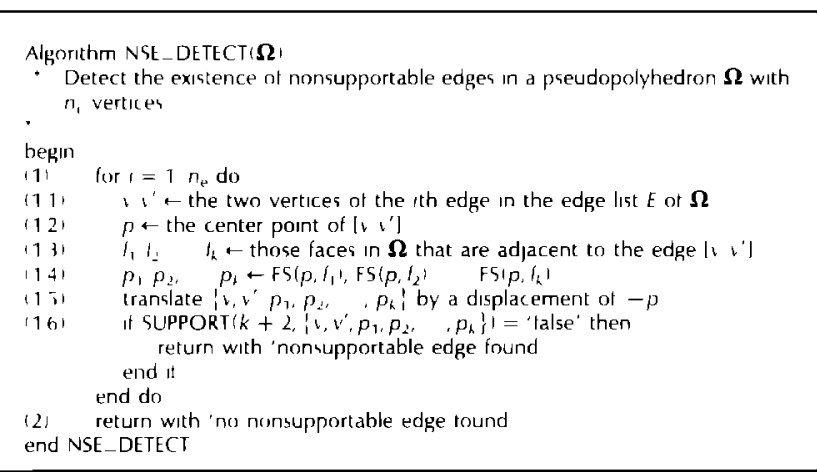

Lemma 7 The existence of nonsupportable vertices and nonsupportable edges of a pseudopolyhedron $\boldsymbol{\Omega}$ with $n_{v}$ vertices, $n_{e}$ edges and $n_{f}$ faces can be detected in at most $O\left(n_{\mathrm{f}}\right)$ tıme

Proof The theorem is proven by showing that both the NSV_DETECT and NSE_DETECT algorithms run in $O\left(n_{f}\right)$ tıme For the NSV_DETECT algorithm, because the SUPPORT procedure runs in linear time, the tıme complexity required by the loop at Step 1 is linear in $\Sigma d_{1}\left(t=1,2, \quad, n_{v}\right)$, where $d_{l}$ is the degree of the $t$ th vertex in $\boldsymbol{\Omega}$, which has been proven to be $U\left(n_{\mathrm{f}}\right)$ in the Appendix of Part 1 of this paper Therefore, NSV_DETECI runs in $O\left(n_{\mathrm{f}}\right)$ time For the NSE_DETECT algorithm, by sımılar reasonıng, the tıme required by the loop at Step 1 is linear in $\Sigma k_{1}\left(r=1,2, \quad, n_{e}\right)$, where $k_{1}$ is the face adjacency index of the ith edge in $\boldsymbol{\Omega}$ In the Appendix of Part 1 of this paper, it has been shown that $\sum k_{1}\left(\imath=1,2, \ldots, n_{\mathrm{e}}\right)$ is $O\left(n_{\mathrm{f}}\right)$ Therefore, NSE_DETECT runs in $O\left(n_{f}\right)$ time

QED

\section{SUMMARY}

It has been established that it takes $O\left(n^{2} \log n\right)$ tıme to determıne if the ASV series of a given $\boldsymbol{\Omega}$ converges in partıcular, it takes $O(n \log n)$ tıme to detect if a deficiency $\boldsymbol{\Omega}$, is nonconvergent To remedy the nonconvergence, an $O(n \log n)$ tıme algorithm is offered to separate the culprit deficiency $\boldsymbol{\Omega}$, into maximally well connected sets

As an expedient alternatıve to the $O\left(n^{2} \log n\right)$ tıme detection for nonconvergence, the sufficiency condition for nonconvergence can be detected in $O(n)$ tıme.

\section{REFERENCES}

1 Woo, T C 'Feature extraction by volume decompositıon' Proc. Cont CAD/CAM Technology in Mechanıcal Engıneerıng Cambridge, MA, USA (1982)

2 Tang, $K$ and Woo, $T$ 'Algorithmic aspects of alternatıng sum of volumes Part 1 Data structure and difference operation' Comput-Aided Des Vol 23 No 5 (1991) pp 357-366

3 Preparata, $\mathbf{F} \mathbf{P}$ and Shamos, $\mathbf{M} \mathbf{L}$ Computational Ceometry Sprınger-Verlag (1985)

4 Chazelle, B M 'Convex decomposition of polyhedra' Proc. ACM Symp Theory of Computing Milwaukee, USA (1981) pp 70-79

5 Requicha, A A G and Voelcker, H B 'Solid modelıng current status and research directions' IEEE Comput Graph \& Applic Vol 3 No 7 (1983) pp 25-37

\section{BIBLIOGRAPHY}

Baer, A, Eastman, $\mathbf{C}$ and Henrion, $\mathbf{M}$ 'Ceometric modelıng a survey' Comput.-Aıded Des Vol 11 No 5 (1979) pp 253-272

Bollobas, C Graph Theory Sprınger-Verlag (1979)

Cary, A 'BUILD users' guide' Document No 102 CAD Group, University of Cambridge, UK (Nov 1979)

Chiyokura, $\mathbf{H}$ and Kimura, F'A method of representıng the solıd design process' IEEE Comput Graph \& Applic Vol 5 No 4 (1985) pp 32-41

Preparata, F P and Hong, S J 'Convex hull of finite sets of points in two and three dimensions' Commun ACM Vol 20 No 2 (1977) pp 87-93 
Requicha, A A G 'Representation for rigid solıds. theory, methods and systems' ACM Comput Surveys Vol 12 No 4 (1980) pp 437-464

Requicha, A A G and Voelcker, H B 'Constructive solid geometry' Production Automation Project TM-25 University of Rochester, USA (Nov 1977)
Weiler, $\mathbf{K}$ 'Topologıcal structures for geometrıc modelıng' PhD Dissertation Rensselaer Polytechnic Institute, USA (Aug 1986)

Woo, T C 'A combinatorial analysis of boundary data structure schemata' IEEE Comput Graph. \& Applic. Vol 5 No 3 (Mar 1985) pp 19-27 